\title{
On the centralizer of diffeomorphisms of the half-line
}

\author{
Hélène Eynard
}

\begin{abstract}
Let $f$ be a smooth diffeomorphism of the half-line fixing only the origin and $Z^{r}$ its centralizer in the group of $C^{r}$ diffeomorphisms. According to well-known results of Szekeres and Kopell, $Z^{1}$ is a one-parameter group. On the other hand, Sergeraert constructed an $f$ whose centralizer $Z^{r}, 2 \leq r \leq \infty$, reduces to the infinite cyclic group generated by $f$. We show that $Z^{r}$ can actually be a proper dense and uncountable subgroup of $Z^{1}$ and that this phenomenon is not scarce.
\end{abstract}

Mathematics Subject Classification (2010). 37E05.

Keywords. Interval diffeomorphism, centralizer, commuting, Liouville number, vector field, flow.

Let $f$ be a smooth diffeomorphism of the closed half-line $\mathbb{R}_{+}$with a single fixed point at the origin. In this article, we study the centralizer of $f$ in the group $D^{r}$ of $C^{r}$ diffeomorphisms of $\mathbb{R}_{+}, 1 \leq r \leq \infty$, that is, the (closed) subgroup $Z_{f}^{r}$ of $D^{r}$ made up of all diffeomorphisms commuting with $f$. The first things to observe are that $Z^{r}$ decreases with $r$, contains the infinite cyclic subgroup generated by $f$ and is quite small. Indeed, for $r=1$, well-known theorems by G. Szekeres and N. Kopell [7], [4] show that $Z_{f}^{1}$ is always a one-parameter subgroup of $D^{1}$ (see also Chapter 4 in [9] and Chapter 4 in [5] for complete proofs and more discussion). For $r \geq 2$, the situation is more subtle, and for instance both of the limit cases permitted by the inclusions

$$
\mathbb{Z} \cong\left\{f^{n}, n \in \mathbb{Z}\right\} \subset Z_{f}^{r} \subset Z_{f}^{1} \cong \mathbb{R}
$$

can occur. According to F. Takens' work [8], if $f$ is not infinitely tangent to the identity at 0 then $Z_{f}^{1}$ consists of smooth diffeomorphisms and therefore coincides with $Z_{f}^{\infty}$. On the other hand, in [6], F. Sergeraert builds a diffeomorphism $f$ whose centralizer $Z_{f}^{2}$ is strictly contained in $Z_{f}^{1}$, and one can actually check [2] that, in this example, $Z_{f}^{2}$ reduces to the group spanned by $f$ - and is hence as small as possible. The following result says that there exist intermediate situations:

Theorem A. There exists a smooth diffeomorphism $f$ of $\mathbb{R}_{+}$with a single fixed point at the origin, whose centralizer $Z_{f}^{r}$, for $2 \leq r \leq \infty$, is a proper, dense and uncountable subgroup of the one-parameter group $Z_{f}^{1}$. 
This theorem follows from the proposition below, where $f$ is the flow at time one of the vector field $\xi$ coming out:

Proposition 1. There exists a complete $C^{1}$ vector field $\xi$ on $\mathbb{R}_{+}$, vanishing only at 0 , whose flow $f^{t}$ at time $t$ is not $C^{2}$ at 0 for $t=1 / 2$ but is smooth on $\mathbb{R}_{+}$for all $t \in \mathbb{Z} \oplus \sum_{\tau \in K} \tau \mathbb{Z}$, where $K \subset \mathbb{R} \backslash \mathbb{Q}$ is a Cantor set.

A natural question to ask now is whether diffeomorphisms $f$ whose centralizer $Z_{f}^{r}, r \geq 2$, is neither the one-parameter group generated by $f$ (namely, $Z_{f}^{1} \cong \mathbb{R}$ ) nor the discrete group spanned by $f$ (that is, $\left\{f^{n}, n \in \mathbb{Z}\right\} \cong \mathbb{Z}$ ), are very peculiar or not. At the end of the paper, Theorem $B$ gives a partial answer to this question: every diffeomorphism of $\mathbb{R}_{+}$which satisfies a certain oscillation condition and belongs to a smooth flow (with the usual hypotheses on the unique fixed point) can be approximated in a suitable sense by diffeomorphisms $f$ whose centralizer $Z_{f}^{r}$ is as in Theorem A. The proof of this second theorem is very similar to that of the first one but involves more technicalities. For this reason, we discuss the weaker statement in priority.

It would also be interesting to know whether the centralizer $Z_{f}^{r}$, when it is a proper subgroup of $\mathbb{R} \cong Z_{f}^{1}$, can contain any Diophantine number. It turns out [2] that the Cantor set we construct in our proof of Proposition 1 contains only Liouville numbers.

Acknowledgements. I am extremely grateful to Sylvain Crovisier for explaining the method of approximation by conjugation to me and suggesting that it could be used in this work to preserve and control the desired smoothness of the limit flow. More generally I am deeply thankful for his continued interest in my progress and his useful comments on this article. I would also like to thank Jean-Christophe Yoccoz for sharing his insight on the subject with me and encouraging me to work on this particular question. These two interactions were possible thanks to the financial support of the Agence Nationale de la Recherche (through the "Symplexe" project). Last but not least, this work would not have been possible without the considerable help of Emmanuel Giroux, who dedicated much of his time and energy to me through countless discussions, reflexions and rewritings, always leading to a better understanding, and I warmly thank him for his uncommon involvement and patience.

\section{Proof of Proposition 1}

1. Overview. The following proof combines the strategy used by F. Sergeraert in [6], Section 4, with the method of approximation by conjugation introduced by D. Anosov and A. Katok in [1] and later developped by many authors (see [3] and references therein). We start with a particular smooth vector field $\xi_{0}$ (the same as in [6]) and build $\xi$ as the limit of a sequence of deformations $\xi_{k}$ where each $\xi_{k}$ is the pullback 
$h_{k}^{*} \xi_{0}$ of $\xi_{0}$ by a smooth diffeomorphism $h_{k}$. Thus, the flow $f_{k}^{t}$ of $\xi_{k}$ is related to the flow $f_{0}^{t}$ of $\xi_{0}$ by $f_{k}^{t}=h_{k}^{-1} \circ f_{0}^{t} \circ h_{k}$. The point is to cook up the conjugations $h_{k}$ so that the diffeomorphisms $f_{k}^{t}, k \geq 1$, converge in the $C^{\infty}$ topology for a dense set of times $t$ but converge only in the $C^{1}$ topology for some other time. In particular, the diffeomorphisms $h_{k}$ diverge in the $C^{2}$ topology. Here, the behaviour of the initial vector field plays a crucial role: we take a vector field $\xi_{0}$ presenting plateaux which accumulate at the origin and whose heights tend to zero but with wild oscillations. According to a theorem of F. Sergeraert [6], Section 3, these oscillations are necessary if we want to create a non-smooth flow with small perturbations $h_{k}$ of the identity. Furthermore, Theorem B at the end of this paper states an oscillation condition which is sufficient for our construction to work.

Let us indicate now how these oscillations come into play. First of all, we pick an initial vector field $\xi_{0}$ vanishing only at the origin, and contracting: every point is attracted by 0 in the future. Or, in other words, the function $\xi_{0} / \partial_{x}$ is negative away from 0 . The graph of this function can then be depicted as an undersea landscape consisting of a sequence of alternating lowlands $L_{k}$ and highlands $H_{k}$ whose respective altitudes $-v_{k}$ and $-u_{k}$ (measured from the water surface, so that $0<u_{k}<v_{k}$ ) go to zero when $k$ grows, but "oscillate wildly" in the sense that the ratios $v_{k} / u_{k}$ tend to infinity.

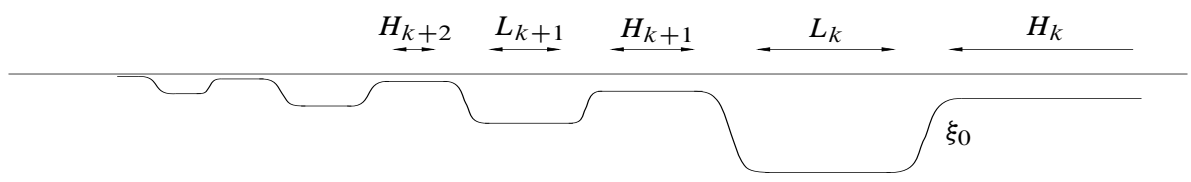

A consequence of this behaviour is that, if an element $f_{0}^{t}$ of the flow takes a segment $S \subset H_{k}$ into $L_{k}$ for some large $k$, then its restriction to $S$ is an affine map with big dilation factor $v_{k} / u_{k}$.

In our deformation process, the diffeomorphisms $h_{k}$ are defined inductively and all coincide with the identity near 0 . Each new perturbation is described by the diffeomorphism $g_{k}=h_{k} \circ h_{k-1}^{-1}$ and its role is to modify the flow of $\xi_{0}$ locally at a specific time $1 / q_{k}$, in a fundamental segment $S_{k}$ of $f_{0}^{1 / q_{k}}$ lying in the lowland $L_{k}$. In other words, $g_{k}^{-1} \circ f_{0}^{1 / q_{k}} \circ g_{k}$ agrees with $f_{0}^{1 / q_{k}}$ outside $S_{k}$. Furthermore, we take $g_{k}$ close enough to the identity so that the $C^{k}$ norms of the maps

and also

$$
g_{k}^{-1} \circ f_{0}^{t} \circ g_{k}-f_{0}^{t}, \quad t \in \frac{1}{q_{k}} \mathbb{Z} \cap[0,1],
$$

$$
h_{k}^{-1} \circ f_{0}^{t} \circ h_{k}-h_{k-1}^{-1} \circ f_{0}^{t} \circ h_{k-1},
$$

are all strictly bounded by $2^{-k}$, and we denote by $I_{k}$ a compact neighbourhood of $\frac{1}{q_{k}} \mathbb{Z} \cap(0,1)$ such that the non-strict bounds still hold for all $t \in I_{k}$. With a suitable 
choice of the sequence $q_{k}$, we can arrange that the intersection of the compact sets $I_{k}$ is a Cantor set $K$ consisting of irrational times $t$ for which the diffeomorphisms $h_{k}^{-1} \circ f_{0}^{t} \circ h_{k}, k \geq 1$, converge in the $C^{\infty}$ topology. Indeed, it suffices to pick $q_{k}$ at each step in such a way that $\frac{1}{q_{k}} \mathbb{Z}$ meets any component of $I_{k-1}$ in at least two points, and also avoids the $k^{\text {th }}$ rational number (for an arbitrary enumeration of $\mathbb{Q}$ ) so that $K=\bigcap I_{k}$ has no rational point.

Although the action of the perturbation diffeomorphism $g_{k}$ on the map $f_{0}^{1 / q_{k}}$ is local, its action on the vector field $\xi_{0}$ and on general elements of its flow is not at all. To see this, let us consider the difference $v_{k}=g_{k}^{*} \xi_{0}-\xi_{0}$. Since $g_{k}$ commutes with $f_{0}^{1 / q_{k}}$ out of $S_{k}$ and coincides with the identity near 0 , it is actually the identity on the whole interval $\left[0, \min S_{k}\right]$. In particular, $v_{k}$ vanishes identically there. Inside $S_{k}$, our choice of $g_{k}$ gives $v_{k}$ the following shape of a $C^{k}$-small wave:

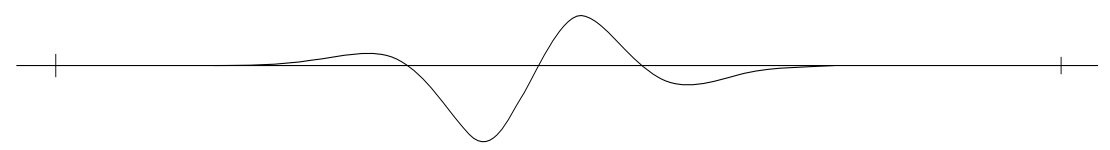

On the other hand, the half-line $\left[\max S_{k},+\infty\right)$ is tiled by the segments $S_{k}^{p}=$ $f_{0}^{-p / q_{k}}\left(S_{k}\right), p \geq 1$. The commutation property noted above now implies that, for every $p \geq 1$,

$$
v_{k \mid S_{k}^{p}}=\left(f_{0}^{p / q_{k}}\right)^{*}\left(v_{k \mid S_{k}}\right) .
$$

In other words, the wave $v_{k \mid S_{k}}$ is propagated to the right of $S_{k}$ by the iterates of $f_{0}^{1 / q_{k}}$. Let us look at the wave $v_{k \mid S_{k}^{p}}$ when $S_{k}^{p}$ sits on the highland $H_{k}$. As explained before, the restriction of $f_{0}^{p / q_{k}}$ to $S_{k}^{p}$ for such a $p$ is an affine map of the form

$$
x \in S_{k}^{p} \mapsto \frac{v_{k}}{u_{k}} x+c_{k} \quad \text { for some } c_{k} \in \mathbb{R} .
$$

Then, according to (1),

$$
\left(v_{k \mid S_{k}^{p}}\right)(x)=\frac{\left(v_{k \mid S_{k}}\right)\left(f_{0}^{p / q_{k}}(x)\right)}{D f_{0}^{p / q_{k}}(x)}=\left(\frac{v_{k}}{u_{k}}\right)^{-1}\left(v_{k \mid S_{k}}\right)\left(\frac{v_{k}}{u_{k}} x+c_{k}\right),
$$

and so, for any integer $m \geq 1$,

$$
D^{m}\left(v_{k \mid S_{k}^{p}}\right)(x)=\left(\frac{v_{k}}{u_{k}}\right)^{m-1} D^{m}\left(v_{k} \mid S_{k}\right)\left(\frac{v_{k}}{u_{k}} x+c_{k}\right) .
$$

Thus, in the course of the propagation, the wave remains $C^{1}$ small but its higher order derivatives are amplified and can become big. As we already said, the difficulty is then to adjust the perturbation diffeomorphisms $g_{k}$ so that the differences $h_{k}^{*} \xi_{0}-\xi_{0}$ 
(which are essentially the superpositions of the propagated waves $v_{l}, l \leq k$ ) diverge in the $C^{2}$ topology while the conjugates $h_{k}^{-1} \circ f_{0}^{t} \circ h_{k}$, for $t$ in the Cantor set $K$, still converge in the $C^{\infty}$ topology. Following Sergeraert, a solution is roughly to take $u_{k}$ and $v_{k}$ respectively equal to $2^{-k^{4}}$ and $2^{-k^{2}}$, while the size of the wave $v_{k} \mid S_{k}$ is defined as $2^{-k^{3}}$.

2. Notation and toolbox. In this short section, we assume that all necessary conditions are met so that the expressions we write make sense. For any $C^{k}$ map $g$ defined on an interval $I \subset \mathbb{R}$ (open or closed), we set

$$
\|g\|_{k}=\sup \left\{\left|D^{m} g(x)\right|, 0 \leq m \leq k, x \in I\right\} \in[0,+\infty] .
$$

If $f: I \rightarrow f(I)$ is an orientation-preserving $C^{2}$ diffeomorphism, we define $L f$ to be

$$
L f=D \log D f=\frac{D^{2} f}{D f} .
$$

The non-linear differential operator $L$ satisfies the following chain rule:

$$
L(h \circ g)=L h \circ g \cdot D g+L g .
$$

To compute higher order derivatives of compositions, we will also use Faà di Bruno's formula in the form

$$
D^{m}(h \circ g)=\sum_{\pi \in \Pi_{m}}\left(D^{|\pi|} h\right) \circ g \cdot \prod_{B \in \pi} D^{|B|} g,
$$

where $\Pi_{m}$ is the set of all partitions $\pi$ of $\{1, \ldots, m\}$ and $|X|$, for any finite set $X$, is the number of its elements.

Let $\eta$ be a vector field on an interval $J$. Throughout the paper, we will make no difference between $\eta$ and the function $\eta / \partial_{x}$, where $x$ is the underlying coordinate in $J$, and in particular we will identify $\partial_{x}$ with 1 . If $J$ is both the source of $g$ and the target of $h$ (where $g$ and $h$ are diffeomorphisms), we can define two new vector fields, $g_{*} \eta$ and $h^{*} \eta$, which are the pushforward of $\eta$ by $g$ and its pullback by $h$, respectively. Viewed as functions, these vector fields are given by

$$
\begin{aligned}
& g_{*} \eta=D g \circ g^{-1} \cdot \eta \circ g^{-1}, \\
& h^{*} \eta=\frac{\eta \circ h}{D h}
\end{aligned}
$$

and so we easily get the following expressions for the derivatives:

$$
\begin{aligned}
& D\left(g_{*} \eta\right)=D \eta \circ g^{-1}+L g \circ g^{-1} \cdot \eta \circ g^{-1}, \\
& D\left(h^{*} \eta\right)=D \eta \circ h-\frac{D^{2} h}{(D h)^{2}} \eta \circ h .
\end{aligned}
$$


3. The initial vector field. The construction involves two smooth functions $\alpha$, $\beta: \mathbb{R} \rightarrow[0,1]$ satisfying the following conditions:

- $\alpha(x)$ equals 0 if $x \leq 1 / 6$ and 1 if $x \geq 1 / 3$;

- $\beta(x)$ equals 0 if $x \leq 1 / 6$ or $x \geq 5 / 6$, and 1 if $1 / 3 \leq x \leq 2 / 3$;
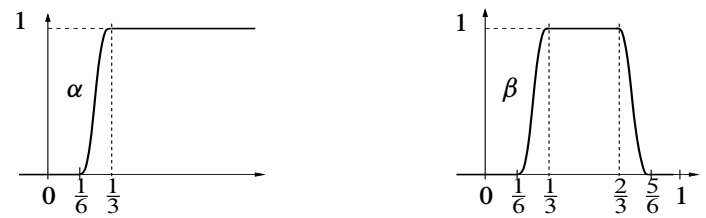

Now, setting $u_{n}=2^{-n^{4}}$ and $v_{n}=2^{-n^{2}}$, we define the vector field $\xi_{0}$ as in [6] by

$$
\begin{gathered}
\xi_{0}(x)=-u_{n+1}-\left(u_{n}-u_{n+1}\right) \alpha\left(2^{n+1} x-1\right)-\left(v_{n}-u_{n}\right) \beta\left(2^{n+1} x-1\right) \\
\text { for } x \in\left[2^{-n-1}, 2^{-n}\right], \quad \xi_{0}(0)=0 \quad \text { and } \quad \xi_{0}(x)=-1 \quad \text { for } x \geq 1 .
\end{gathered}
$$

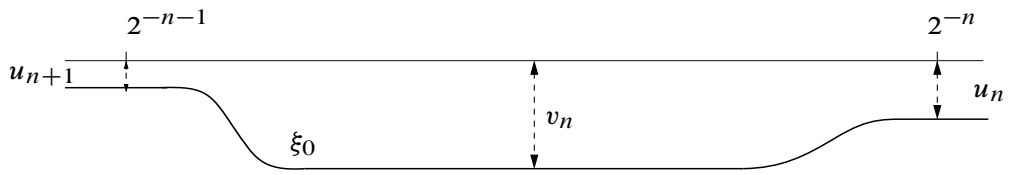

From now on, we denote by $\left\{f_{0}^{t}, t \in \mathbb{R}\right\}$ the flow of $\xi_{0}$ and by $\psi: \mathbb{R} \rightarrow \mathbb{R}_{+}^{*}$ the diffeomorphism given by $\psi(t)=f_{0}^{t}(1)$ for all $t \in \mathbb{R}$. Note that, since $D \psi=\xi_{0} \circ \psi$,

$$
\xi_{0}=D \psi \circ \psi^{-1} \quad \text { and } \quad D \xi_{0}=L \psi \circ \psi^{-1} .
$$

We also fix a forward orbit $\left\{a_{l}, l \geq 0\right\}$ of $f_{0}=f_{0}^{1}$, where $a_{0}=1$ and $a_{l}=f_{0}\left(a_{l-1}\right)$ $=\psi(l)$ for all $l \geq 1$.

One easily checks that $\xi_{0}$ is smooth, contracting, infinitely flat at the origin and $C^{1}$-bounded - with $1<\left\|\xi_{0}\right\|_{1}<+\infty$. Furthermore, $\xi_{0}$ equals $-v_{n}$ identically on the central third of $\left[2^{-n-1}, 2^{-n}\right]$, namely $\left[2^{-n-1}+2^{-n-1} / 3,2^{-n}-2^{-n-1} / 3\right]$, and $-u_{n}$ on $\left[2^{-n}-2^{-n-1} / 6,2^{-n}+2^{-n} / 6\right]$. A simple computation of travel time at constant speed shows that for all $n \geq 4$, there exist integers $i(n)$ and $j(n)$ such that

$$
2^{-n}-\frac{1}{6} 2^{-n-1} \leq a_{i(n)+2}<a_{i(n)-1} \leq 2^{-n}+\frac{1}{6} 2^{-n}
$$

and

$$
2^{-n-1}+\frac{1}{3} 2^{-n-1} \leq a_{j(n)+2}<a_{j(n)-1} \leq 2^{-n}-\frac{1}{3} 2^{-n-1} .
$$

Thus $\xi_{0}$ equals $-v_{n}$ on $\left[a_{j(n)+2}, a_{j(n)-1}\right]$, and hence $f_{0}^{t}$ induces on $\left[a_{j(n)+1}, a_{j(n)-1}\right]$ the translation by $-t v_{n}$ for $0 \leq t \leq 1$. Similarly, $f_{0}^{t}$ induces the translation by $-t u_{n}$ in a neighbourhood of $a_{i(n)}$. 
4. The deformation process. Our goal is now to produce a sequence $h_{k}$ of smooth diffeomorphisms of $\mathbb{R}_{+}$such that the vector fields $\xi_{k}=h_{k}^{*} \xi_{0}$ converge in the $C^{1}$ topology to the vector field $\xi$ of Proposition 1. In order to have regular perturbation patterns (and easier computations), we actually work at time scale, i.e. we define $h_{k}$ as the conjugate $\psi \circ \Phi_{k} \circ \psi^{-1}$ of a smooth diffeomorphism $\Phi_{k}$ of $\mathbb{R}$ (which coincides with the identity near $+\infty$ so that $h_{k}$ is also the identity near 0 ). Conforming to the general scheme of the approximation by conjugation method (see [3]), $\Phi_{k}$ is obtained as a composition

$$
\Phi_{k}=\varphi_{k} \circ \varphi_{k-1} \circ \cdots \circ \varphi_{1}
$$

where the diffeomorphisms $\varphi_{k}$ are manufactured inductively from a fixed function $\gamma$ and two adjustment integer parameters $q_{k}$ and $n_{k}$. The details of the construction follow.

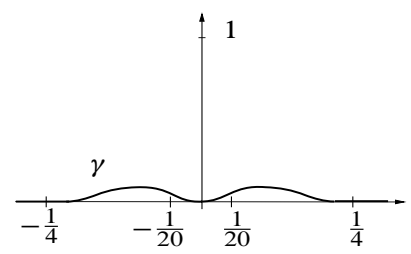

Let $\gamma: \mathbb{R} \rightarrow[0,1]$ be a smooth function supported in $[-1 / 4,1 / 4]$ and satisfying $\gamma(t)=t^{2} / 2$ around 0 . Given positive integers $q, n$, set $w_{n}=2^{-n^{3}}$ and denote by $\gamma_{q, n}: \mathbb{R} \rightarrow[0,1]$ the smooth function defined by

$$
\gamma_{q, n}(t)=w_{n} \gamma(q(t-j(n))) \quad \text { for all } t \in \mathbb{R} .
$$

Clearly, $\gamma_{q, n}$ is supported in $\left[j(n)-\frac{1}{4 q}, j(n)+\frac{1}{4 q}\right]$. Moreover, for every integer $m \geq 1$ and all $t \in \mathbb{R}$,

$$
D^{m} \gamma_{q, n}(t)=w_{n} q^{m} D^{m} \gamma(q(t-j(n)))
$$

and hence

$$
\left\|\gamma_{q, n}\right\|_{m}=w_{n} q^{m}\|\gamma\|_{m}
$$

In particular, by taking $n$ large compared to $q$ once $m$ is fixed, one can make the $C^{m}$ norm of $\gamma_{q, n}$ arbitrarily small.

Now let $J_{q, n}$ be the interval $\left[j(n)-\frac{1}{2 q}, j(n)+\frac{1}{2 q}\right]$ and define $\varphi_{q, n}: \mathbb{R} \rightarrow \mathbb{R}$ as the map meeting the following properties:

- $\varphi_{q, n}(t)=t$ for $t>j(n)+\frac{1}{2 q}$;

- $\varphi_{q, n}(t)=t+\gamma_{q, n}(t)$ for $t \in J_{q, n}$;

- $\varphi_{q, n}$ commutes with the translation by $\frac{1}{q}$ outside $J_{q, n}$, and so

$$
\varphi_{q, n}(t)=t+\gamma_{q, n}\left(t+\frac{p}{q}\right) \quad \text { if } t \in\left(J_{q, n}-\frac{p}{q}\right), p \geq 0
$$


In short, we can write

$$
\varphi_{q, n}(t)=t+\sum_{p \geq 0} \gamma_{q, n}\left(t+\frac{p}{q}\right) \quad \text { for all } t \in \mathbb{R},
$$

and similarly,

$$
\begin{aligned}
D \varphi_{q, n}(t) & =1+\sum_{p \geq 0} D \gamma_{q, n}\left(t+\frac{p}{q}\right), \\
D^{m} \varphi_{q, n}(t) & =\sum_{p \geq 0} D^{m} \gamma_{q, n}\left(t+\frac{p}{q}\right) \quad \text { for all } m \geq 2 .
\end{aligned}
$$

Note that for every $t \in \mathbb{R}$, at most one term in each sum is nonzero since the support of $\gamma_{q, n}$ has length less than $1 / q$. These equations imply that

$$
\left\|\varphi_{q, n}-\mathrm{id}\right\|_{m}=\left\|\gamma_{q, n}\right\|_{m}
$$

and, in particular, $\varphi_{q, n}$ is a diffeomorphism provided $\left\|\gamma_{q, n}\right\|_{1}<1$.

The following lemma will be used later (in the proof of Lemma 4) to show that the limit flow coming out of our construction is not smooth at time $1 / 2$ :

Lemma 2. For all $l \geq 1$, let $q_{l}$ and $n_{l}$ be positive integers with $q_{l}$ odd and $w_{n_{l}} q_{l}\|\gamma\|_{1}<1$. Then for every $k \geq 1$ the diffeomorphism $\Phi_{k}$ defined by

$$
\Phi_{k}=\varphi_{k} \circ \varphi_{k-1} \circ \cdots \circ \varphi_{1}, \quad \text { where } \varphi_{l}=\varphi_{q_{l}, n_{l}} \text {, }
$$

has the following behaviour on $\frac{1}{2} \mathbb{Z}$ :

- $\Phi_{k}$ coincides with the identity in a neighbourhood of $\mathbb{Z}+\frac{1}{2}$;

- $\Phi_{k}$ is tangent to the identity on $\mathbb{Z}-$ meaning that $\Phi_{k}(l)=l$ and $D \Phi_{k}(l)=1$ for all $l \in \mathbb{Z}$

- $\left(L \Phi_{k}-L \Phi_{k-1}\right)(l)$, for $l \in \mathbb{Z}$, equals $w_{n_{k}} q_{k}^{2}$ if $l \leq j\left(n_{k}\right)$ and 0 otherwise.

Proof. Since $\gamma_{l}=\gamma_{q_{l}, n_{l}}$ is supported in $\left[-\frac{1}{4 q_{l}}, \frac{1}{4 q_{l}}\right]+j\left(n_{l}\right)$ and

$$
\varphi_{l}=\mathrm{id}+\sum_{p \geq 0} \gamma_{l}\left(t+\frac{p}{q_{l}}\right)
$$

$\varphi_{l}$ is the identity on the $\frac{1}{4 q_{l}}$-neighbourhood of $\frac{1}{q_{l}} \mathbb{Z}+\frac{1}{2 q_{l}}$. But $q_{l}$ is odd, say $q_{l}=$ $2 s_{l}+1$, so

$$
\frac{1}{2}=\frac{q_{l}}{2 q_{l}}=\frac{2 s_{l}+1}{2 q_{l}}=\frac{s_{l}}{q_{l}}+\frac{1}{2 q_{l}} \in \frac{1}{q_{l}} \mathbb{Z}+\frac{1}{2 q_{l}},
$$


and hence $\frac{1}{q_{l}} \mathbb{Z}+\frac{1}{2 q_{l}}$ contains $\mathbb{Z}+\frac{1}{2}$ for all $l \geq 1$. Therefore $\Phi_{k}=\varphi_{k} \circ \cdots \circ \varphi_{1}$ is the identity in a neighbourhood of $\mathbb{Z}+\frac{1}{2}$. On the other hand, since $\gamma(0)=D \gamma(0)=0$, each $\varphi_{l}$ is $C^{1}$ tangent to the identity on $\frac{1}{q_{l}} \mathbb{Z} \supset \mathbb{Z}$, so $\Phi_{k}$ is $C^{1}$ tangent to the identity on $\mathbb{Z}$.

Now, applying the chain rule (2) for the operator $L=D^{2} / D$ to $\Phi_{k}=\varphi_{k} \circ \Phi_{k-1}$, we get

$$
L \Phi_{k}=L \varphi_{k} \circ \Phi_{k-1} \cdot D \Phi_{k-1}+L \Phi_{k-1} .
$$

For $l \in \mathbb{Z}$, we have seen above that $\Phi_{k-1}(l)=l$ and $D \Phi_{k-1}(l)=1$, so

$$
\left(L \Phi_{k}-L \Phi_{k-1}\right)(l)=L \varphi_{k}(l) .
$$

If $l>j\left(n_{k}\right)$ then $L \varphi_{k}(l)=0$ just because $\varphi_{k}$ agrees with the identity on the interval $\left[j\left(n_{k}\right)+\frac{1}{2 q_{k}},+\infty\right)$. If $l \leq j\left(n_{k}\right)$ then (11) and (12) give

$$
L \varphi_{k}(l)=\frac{D^{2} \varphi_{k}(l)}{D \varphi_{k}(l)}=D^{2} \varphi_{k}(l)=D^{2} \gamma_{k}\left(j\left(n_{k}\right)\right)=w_{n_{k}} q_{k}^{2},
$$

which completes the proof.

For the next lemma, we fix an enumeration of the rational numbers, $\mathbb{Q}=\left\{r_{k}\right\}_{k \geq 1}$, and set $\Phi_{0}=$ id and $I_{0}=[0,1]$. Moreover, as in Lemma 2, we will henceforth abbreviate $\varphi_{q_{l}, n_{l}}$ as $\varphi_{l}$ (and similarly $\gamma_{q_{l}, n_{l}}$ as $\gamma_{l}$ and $J_{q_{l}, n_{l}}$ as $J_{l}$ ).

Lemma 3. For suitably chosen increasing sequences of positive integers $q_{k}$ and $n_{k}$, the diffeomorphisms $\Phi_{k}=\varphi_{k} \circ \cdots \circ \varphi_{1}$ and $h_{k}=\psi \circ \Phi_{k} \circ \psi^{-1}$, the vector fields $\xi_{k}=h_{k}^{*} \xi_{0}$ and their flows $f_{k}^{t}$ satisfy the following estimates for every $k \geq 1$ :

$$
\begin{aligned}
& \left\|\Phi_{k}-\Phi_{k-1}\right\|_{k+1} \leq 2^{-k-1}, \\
& \left\|\xi_{k}-\xi_{k-1}\right\|_{1} \leq 2^{-k}, \\
& \left\|\left.\left(f_{k}^{t}-f_{k-1}^{t}\right)\right|_{[0,1]}\right\|_{k} \leq 2^{-k} \text { for all } t \in I_{k} \cup\{1\},
\end{aligned}
$$

where $I_{k} \subset I_{k-1}$ is a compact set avoiding the $k^{\text {th }}$ rational number $r_{k}$ and consisting of $2^{k}$ disjoint segments of nonzero length, two in each component of $I_{k-1}$.

Proof. Let $k \geq 1$ and assume we already chose $q_{l}$ and $n_{l}$ for $1 \leq l \leq k-1$ in such a way that estimates $\left(\mathrm{i}_{l}\right),\left(\mathrm{ii}_{l}\right)$ and $\left(\mathrm{iii}_{l}\right)$ hold. In particular, since $\Phi_{0}=\mathrm{id}$ by convention,

$$
\left\|\Phi_{k-1}-\mathrm{id}\right\|_{2} \leq \sum_{l=1}^{k-1}\left\|\Phi_{l}-\Phi_{l-1}\right\|_{2} \leq \sum_{l=1}^{k-1} 2^{-l-1}=\frac{1}{2}-2^{-k} \leq \frac{1}{2} .
$$


Take an odd integer $q_{k}>q_{k-1}$ such that $\frac{1}{q_{k}} \mathbb{Z}$ avoids $r_{k}$ and meets the interior of each component of $I_{k-1}$ in at least two points. Then pick $n_{k}>n_{k-1}$ such that

$$
\left\|\gamma_{k}\right\|_{k+1} \leq \frac{2^{-k-4} v_{n_{k}}^{k-1}}{\left|\Pi_{k+1}\right|\left\|D \Phi_{k-1}\right\|_{k}^{k+1}\left\|\xi_{0}\right\|_{1}}
$$

i.e.

$$
\frac{w_{n_{k}}}{v_{n_{k}}^{k-1}} \leq \frac{2^{-k-4} q_{k}^{-k-1}}{\left|\Pi_{k+1}\right|\|\gamma\|_{k+1}\left\|D \Phi_{k-1}\right\|_{k}^{k+1}\left\|\xi_{0}\right\|_{1}},
$$

which is possible since

$$
\frac{w_{n}}{v_{n}^{k-1}}=2^{-n^{3}+(k-1) n^{2}}=\mathrm{o}(1) .
$$

Note that inequality (15) clearly implies $\left\|\gamma_{k}\right\|_{1}<1$, and so $\varphi_{k}$ is a diffeomorphism (remember that $\left\|\varphi_{k}-\mathrm{id}\right\|_{m}=\left\|\gamma_{k}\right\|_{m}$ ).

Let us first prove that this choice of $n_{k}$ implies ( $\left.i_{k}\right)$. Since $\Phi_{k}=\varphi_{k} \circ \Phi_{k-1}$, Faà di Bruno's formula (3) gives, for $0 \leq m \leq k+1$,

$$
D^{m}\left(\Phi_{k}-\Phi_{k-1}\right)=\sum_{\pi \in \Pi_{m}} D^{|\pi|}\left(\varphi_{k}-\mathrm{id}\right) \circ \Phi_{k-1} \cdot \prod_{B \in \pi} D^{|B|} \Phi_{k-1} .
$$

But for every partition $\pi \in \Pi_{m}$ with $m \leq k+1$,

$$
\left\|D^{|\pi|}\left(\varphi_{k}-\mathrm{id}\right) \circ \Phi_{k-1}\right\|_{0}=\left\|\gamma_{k}\right\|_{|\pi|} \leq\left\|\gamma_{k}\right\|_{k+1}
$$

and

$$
\prod_{B \in \pi}\left|D^{|B|} \Phi_{k-1}\right| \leq\left\|D \Phi_{k-1}\right\|_{k}^{k+1}
$$

and so

$$
\left\|\Phi_{k}-\Phi_{k-1}\right\|_{k+1} \leq\left|\Pi_{k+1}\right|\left\|\gamma_{k}\right\|_{k+1}\left\|D \Phi_{k-1}\right\|_{k}^{k+1} .
$$

Thus, by the choice of $n_{k}$ in (15),

$$
\left\|\Phi_{k}-\Phi_{k-1}\right\|_{k+1} \leq \frac{2^{-k-4} v_{n_{k}}^{k-1}}{\left\|\xi_{0}\right\|_{1}} \leq 2^{-k-1},
$$

which is the desired estimate $\left(i_{k}\right)$ (note that $\left\|\xi_{0}\right\|_{1} \geq 1$ ).

To prove $\left(\mathrm{ii}_{k}\right)$, let us define

$$
\eta_{k}=\Phi_{k}^{*} \partial_{t}-\Phi_{k-1}^{*} \partial_{t} \quad \text { and } \quad \zeta_{k}=\varphi_{k}^{*} \partial_{t}-\partial_{t},
$$

so that

$$
\eta_{k}=\Phi_{k-1}^{*} \zeta_{k} \quad \text { and } \quad \xi_{k}-\xi_{k-1}=\psi_{*} \eta_{k}
$$


Viewing $\zeta_{k}$ as a function,

$$
\zeta_{k}=\frac{1}{D \varphi_{k}}-1 \quad \text { and } \quad D \zeta_{k}=-\frac{D^{2} \varphi_{k}}{\left(D \varphi_{k}\right)^{2}} .
$$

Given the choice of $n_{k}$ in (15),

$$
\left\|D \varphi_{k}-1\right\|_{0}=\left\|D \gamma_{k}\right\|_{0} \leq 2^{-k-4}\left\|\xi_{0}\right\|_{1}^{-1} \quad\left(\text { and so }\left\|\frac{1}{D \varphi_{k}}\right\|_{0} \leq 2\right),
$$

and

$$
\left\|D^{2} \varphi_{k}\right\|_{0}=\left\|D^{2} \gamma_{k}\right\|_{0} \leq 2^{-k-4}\left\|\xi_{0}\right\|_{1}^{-1},
$$

so

$$
\left|\zeta_{k}\right| \leq 2^{-k-3}\left\|\xi_{0}\right\|_{1}^{-1} \quad \text { and } \quad\left|D \zeta_{k}\right| \leq 2^{-k-2}\left\|\xi_{0}\right\|_{1}^{-1} .
$$

Next, applying (5) and (7) to $\eta_{k}=\Phi_{k-1}^{*} \zeta_{k}$,

$$
\eta_{k}=\frac{\zeta_{k} \circ \Phi_{k-1}}{D \Phi_{k-1}} \quad \text { and } \quad D \eta_{k}=D \zeta_{k} \circ \Phi_{k-1}-\frac{D^{2} \Phi_{k-1}}{\left(D \Phi_{k-1}\right)^{2}} \zeta_{k} \circ \Phi_{k-1}
$$

so, according to (14) and (16),

$$
\begin{aligned}
\left|\eta_{k}\right| & \leq 2^{-k-2}\left\|\xi_{0}\right\|_{1}^{-1}, \\
\left|D \eta_{k}\right| & \leq 2^{-k-2}\left\|\xi_{0}\right\|_{1}^{-1}+\frac{4}{2} 2^{-k-3}\left\|\xi_{0}\right\|_{1}^{-1}=2^{-k-1}\left\|\xi_{0}\right\|_{1}^{-1}
\end{aligned}
$$

Now, applying (4), (6) and (8) to $\xi_{k}-\xi_{k-1}=\psi_{*} \eta_{k}$,

$$
\begin{aligned}
\left|\xi_{k}-\xi_{k-1}\right| & =\left|\eta_{k} \circ \psi^{-1} \cdot \xi_{0}\right| \leq\left\|\eta_{k}\right\|_{0}\left\|\xi_{0}\right\|_{0} \leq 2^{-k-2}, \\
\left|D\left(\xi_{k}-\xi_{k-1}\right)\right| & =\left|D \eta_{k} \circ \psi^{-1}+D \xi_{0} \cdot \eta_{k} \circ \psi^{-1}\right| \\
& \leq 2\left\|\eta_{k}\right\|_{1}\left\|\xi_{0}\right\|_{1} \leq 2^{-k}
\end{aligned}
$$

Thus, $\left\|\xi_{k}-\xi_{k-1}\right\|_{1} \leq 2^{-k}$ as stated in estimate ( $\mathrm{ii}_{k}$ ).

Let us finally prove $\left(\mathrm{iii}_{k}\right)$. Set $\varphi_{0}=\mathrm{id}$ and denote by $\sigma_{l}^{t}$ the flow of $\varphi_{l}^{*} \partial_{t}$ for $0 \leq l \leq k$. Then $\sigma_{0}^{t}$ is just the translation by $t$ and

$$
\sigma_{k}^{t}=\varphi_{k}^{-1} \circ \sigma_{0}^{t} \circ \varphi_{k}
$$

Since

$$
\xi_{k}=\psi_{*} \Phi_{k}^{*} \partial_{t}=\psi_{*} \Phi_{k-1}^{*} \varphi_{k}^{*} \partial_{t} \quad \text { and } \quad \xi_{k-1}=\psi_{*} \Phi_{k-1}^{*} \partial_{t},
$$

their flows are given by

$$
f_{k}^{t}=\psi \circ \Phi_{k-1}^{-1} \circ \sigma_{k}^{t} \circ \Phi_{k-1} \circ \psi^{-1} \text { and } f_{k-1}^{t}=\psi \circ \Phi_{k-1}^{-1} \circ \sigma_{0}^{t} \circ \Phi_{k-1} \circ \psi^{-1} \text {. }
$$


By definition, $\varphi_{k}=\varphi_{q_{k}, n_{k}}$ commutes with the translation $\sigma_{0}^{1 / q_{k}}$ outside $J_{k}=J_{q_{k}, n_{k}}$. Consequently, $\varphi_{k}$ commutes with any iterate $\sigma_{0}^{p / q_{k}}, p \geq 1$, outside the interval

$$
\left[j\left(n_{k}\right)+\frac{1}{2 q_{k}}-\frac{p}{q_{k}}, j\left(n_{k}\right)+\frac{1}{2 q_{k}}\right]=\bigcup_{q=0}^{p-1}\left(J_{k}-\frac{q}{q_{k}}\right) .
$$

Therefore, $\sigma_{k}^{p / q_{k}}$ equals $\sigma_{0}^{p / q_{k}}$ outside this interval, and in particular, for $0 \leq p \leq q_{k}$, outside

$$
M_{k}=\left[j\left(n_{k}\right)-1+\frac{1}{2 q_{k}}, j\left(n_{k}\right)+\frac{1}{2 q_{k}}\right] .
$$

On the other hand, for $t \in J_{k}$,

$$
\begin{aligned}
& \sigma_{k}^{1 / q_{k}}(t)=\varphi_{k}^{-1}\left(\varphi_{k}(t)+\frac{1}{q_{k}}\right) \\
& =\varphi_{k}^{-1}\left(t+\gamma_{k}(t)+\frac{1}{q_{k}}\right) \quad \text { by definition of } \varphi_{k} \text { on } J_{k} \\
& =t+\frac{1}{q_{k}}+\gamma_{k}(t) \quad \text { because } t+\gamma_{k}(t)+\frac{1}{q_{k}}>j\left(n_{k}\right)+\frac{1}{2 q_{k}} \text {, } \\
& =\sigma_{0}^{1 / q_{k}}(t)+\gamma_{k}(t) .
\end{aligned}
$$

Thus, $\sigma_{k}^{1 / q_{k}}-\sigma_{0}^{1 / q_{k}}=\gamma_{k}$. Similarly, for any $p \geq 1$,

$$
\sigma_{k}^{p / q_{k}}(t)-\sigma_{0}^{p / q_{k}}(t)=\sum_{q=0}^{p-1} \gamma_{k}\left(t+\frac{q}{q_{k}}\right) \text { for all } t \in \mathbb{R}
$$

so

$$
\left\|\sigma_{k}^{p / q_{k}}-\sigma_{0}^{p / q_{k}}\right\|_{m}=\left\|\gamma_{k}\right\|_{m}
$$

(again since at most one term of the sum is nonzero in (17)). Now, in the region $M_{k}$ where $\sigma_{k}^{p / q_{k}}$ and $\sigma_{0}^{p / q_{k}}$ disagree for $0 \leq p \leq q_{k}$, the diffeomorphism $\Phi_{k-1}$ is the identity. Moreover, $\psi\left(j\left(n_{k}\right)\right)=a_{j\left(n_{k}\right)}$ and $\psi\left(M_{k}\right) \subset\left[a_{j\left(n_{k}\right)+1}, a_{j\left(n_{k}\right)-1}\right]$ so, by (10), $\psi$ restricted to $M_{k}$ is an affine map with slope $-v_{n_{k}}$. As a consequence, the derivatives of

$$
f_{k}^{p / q_{k}}=\psi \circ \Phi_{k-1}^{-1} \circ \sigma_{k}^{p / q_{k}} \circ \Phi_{k-1} \circ \psi^{-1}
$$

have a simple expression on $\psi\left(M_{k}\right)$ :

$$
D^{m}\left(f_{k}^{p / q_{k}}\right)=\left(-v_{n_{k}}\right)^{1-m} D^{m}\left(\sigma_{k}^{p / q_{k}}\right) \circ \psi^{-1} .
$$

Similarly, again on $\psi\left(M_{k}\right)$,

$$
D^{m}\left(f_{k-1}^{p / q_{k}}\right)=\left(-v_{n_{k}}\right)^{1-m} D^{m}\left(\sigma_{0}^{p / q_{k}}\right) \circ \psi^{-1} .
$$


Therefore, for $0 \leq p \leq q_{k}$ and $0 \leq m \leq k$,

$$
\begin{aligned}
\left|D^{m}\left(f_{k}^{p / q_{k}}-f_{k-1}^{p / q_{k}}\right)\right| & \leq v_{n_{k}}^{1-m}\left\|\sigma_{k}^{p / q_{k}}-\sigma_{0}^{p / q_{k}}\right\|_{m} \\
& =v_{n_{k}}^{1-m}\left\|\gamma_{k}\right\|_{m} \leq v_{n_{k}}^{1-k}\left\|\gamma_{k}\right\|_{k} \leq 2^{-k-4}
\end{aligned}
$$

according to our choice of $n_{k}$ in (15), and thus

$$
\left\|f_{k}^{t}-f_{k-1}^{t}\right\|_{k} \leq 2^{-k-4} \quad \text { for all } t \in \frac{1}{q_{k}} \mathbb{Z} \cap[0,1] .
$$

Now let $T_{k}$ be a subset of $\frac{1}{q_{k}} \mathbb{Z} \cap I_{k-1}$ with exactly two points in each of the $2^{k-1}$ connected components of $I_{k-1}$ (remember that $q_{k}$ was chosen so that there are at least two points there). Since both vector fields $\xi_{k}$ and $\xi_{k-1}$ are smooth on $\mathbb{R}_{+}$, we can find a compact neighbourhood $I_{k}$ of $T_{k}$ in $I_{k-1} \backslash\left\{r_{k}\right\}$ consisting of $2^{k}$ segments, such that

$$
\left\|\left.\left(f_{k}^{t}-f_{k-1}^{t}\right)\right|_{[0,1]}\right\|_{k} \leq 2^{-k} \text { for all } t \in I_{k} \cup\{1\}
$$

(in fact, the restriction to $[0,1]$ is not essential here: one can prove that the difference $f_{k}^{t}-f_{k-1}^{t}$ is 1 -periodic on $[1, \infty)$, and $I_{k}$ can thus be chosen so that the above bound holds on all of $\mathbb{R}_{+}$). This completes the proof of $\left(\mathrm{iii}_{k}\right)$, and thus of Lemma 3.

\section{The limit vector field}

Lemma 4. The vector fields $\xi_{k}, k \geq 1$, of Lemma 3 converge in the $C^{1}$ topology on $\mathbb{R}_{+}$, and in the $C^{\infty}$ topology on $\mathbb{R}_{+}^{*}$, to a vector field $\xi$ which satisfies all properties stated in Proposition 1 with $K=\bigcap I_{k}$.

Proof. The $C^{1}$ convergence of the vector fields $\xi_{k}$ on $\mathbb{R}_{+}$follows directly from estimate $\left(\mathrm{ii}_{k}\right)$ in Lemma 3 . Next, estimate $\left(\mathrm{i}_{k}\right)$ shows that the diffeomorphisms $\Phi_{k}$ converge in the $C^{\infty}$ topology to a smooth diffeomorphism $\Phi$ of $\mathbb{R}$, so the vector fields $\Phi_{k}^{*} \partial_{t}$ converge in the $C^{\infty}$ topology to $\Phi^{*} \partial_{t}$. Now $\xi_{k}$ equals $\psi_{*} \Phi_{k}^{*} \partial_{t}$ on $\mathbb{R}_{+}^{*}$ and $\psi$ is a smooth diffeomorphism from $\mathbb{R}$ to $\mathbb{R}_{+}^{*}$. Given any compact set $A \subset \mathbb{R}_{+}^{*}$ and any integer $m \geq 0$, the restriction of $\psi$ to $\psi^{-1}(A)$ is $C^{m}$-bounded, and hence the vector fields $\xi_{k}$ converge $C^{m}$ uniformly to $\xi$ on $A$. Therefore, the vector fields $\xi_{k}$ converge to $\xi$ on $\mathbb{R}_{+}^{*}$ in the $C^{\infty}$ (compact-open) topology.

The convergence of the vector fields $\xi_{k}$ implies a similar convergence of their flows $f_{k}^{t}$ to the flow $f^{t}$ of $\xi$. Furthermore, estimate ( $\left(\mathrm{iii}_{k}\right)$ in Lemma 3 shows that, for $t \in K \cup\{1\}$, the restrictions $f_{k \mid[0,1]}^{t}$ converge in the $C^{m}$ topology on $[0,1]$ for any $m \geq 0$. In the end, the diffeomorphisms $f_{k}^{t}, t \in K \cup\{1\}$, converge in the $C^{\infty}$ topology on $\mathbb{R}_{+}$, so $f^{t}$ is smooth for all $t \in K \cup\{1\}$, and hence for all $t \in \mathbb{Z} \oplus \sum_{\tau \in K} \tau \mathbb{Z}$. Note here that each $I_{k}$, by construction, is a compact set avoiding the $k^{\text {th }}$ rational number and consisting of $2^{k}$ segments, two in each component of $I_{k-1}$, so $K=\bigcap I_{k}$ is indeed a Cantor set. 
The last thing we have to prove is that $f^{1 / 2}$ is not $C^{2}$ at 0 or, equivalently, that $L f^{1 / 2}=D^{2} f^{1 / 2} / D f^{1 / 2}$ is not continuous at 0 . Let us compute $L f^{1 / 2}$ at a point $a_{i\left(n_{l}\right)}$, as defined in (9), for $l \in \mathbb{N}$. Taking the limit of the maps

$$
f_{k}^{1 / 2}=\psi \circ \Phi_{k}^{-1} \circ\left(\mathrm{id}+\frac{1}{2}\right) \circ \Phi_{k} \circ \psi^{-1},
$$

we get

$$
f^{1 / 2}=\psi \circ \Phi^{-1} \circ\left(\mathrm{id}+\frac{1}{2}\right) \circ \Phi \circ \psi^{-1} .
$$

Let us set $\sigma=\Phi^{-1} \circ\left(\mathrm{id}+\frac{1}{2}\right) \circ \Phi$, so that $f^{1 / 2}=\psi \circ \sigma \circ \psi^{-1}$. Near $a_{i\left(n_{l}\right)}$, the map $\psi^{-1}$ is affine, with slope $-u_{n_{l}}^{-1}$, so

$$
L f^{1 / 2}\left(a_{i\left(n_{l}\right)}\right)=-\frac{1}{u_{n_{l}}} L \sigma\left(i\left(n_{l}\right)\right) .
$$

On the other hand, by (2) applied twice,

$$
L \sigma\left(i\left(n_{l}\right)\right)=L \Phi^{-1}\left(\Phi\left(i\left(n_{l}\right)\right)+\frac{1}{2}\right) \cdot D \Phi\left(i\left(n_{l}\right)\right)+L \Phi\left(i\left(n_{l}\right)\right) .
$$

According to Lemma 2 , each $\Phi_{k}$, and hence $\Phi$, is tangent to the identity on $\frac{1}{2} \mathbb{Z}$ provided all integers $q_{k}$ were chosen odd. Moreover, $\Phi_{k}$ and $\Phi_{k}^{-1}$ coincide with the identity near $\mathbb{Z}+\frac{1}{2}$, so $L \Phi^{-1}\left(i\left(n_{l}\right)+\frac{1}{2}\right)=0$. Summing up, and using the third property in Lemma 2 , we get

$$
L \sigma\left(i\left(n_{l}\right)\right)=L \Phi\left(i\left(n_{l}\right)\right)=\sum_{k \geq 1}\left(L \Phi_{k}-L \Phi_{k-1}\right)\left(i\left(n_{l}\right)\right)=\sum_{k \geq l} w_{n_{k}} q_{k}^{2} .
$$

Therefore,

$$
L f^{1 / 2}\left(a_{i\left(n_{l}\right)}\right)=-\frac{1}{u_{n_{l}}} \sum_{k \geq l} w_{n_{k}} q_{k}^{2}<-\frac{w_{n_{l}}}{u_{n_{l}}} \rightarrow-\infty
$$

and so $f^{1 / 2}$ is not $C^{2}$ at 0 .

\section{More examples}

Let $S$ denote the space of smooth diffeomorphisms of $\mathbb{R}_{+}$which are infinitely tangent to the identity at the origin and have no other fixed point. We say that a diffeomorphism $f$ of $\mathbb{R}_{+}$is contracting if $f(x)<x$ for all $x>0$, and we call Szekeres vector field of $f$ the unique $C^{1}$ vector field generating the one-parameter group $Z_{f}^{1}$ [7], [4]. 
As mentioned in the introduction, the question we discuss in this section is whether the phenomenon presented in Theorem A is very peculiar or quite general. First of all, because of Takens' work [8], this phenomenon is limited to $S$. A difficulty then is that there is no obviously relevant topology on $S$ for our problem. In particular, the $C^{\infty}$ compact-open topology restricted to $S$ is extremely coarse: given any two diffeomorphisms $f, g \in S$, which are both contracting, say, it is easy to construct a sequence of diffeomorphisms $f_{k} \in S$ which converge to $f$ in the $C^{\infty}$ topology and whose germs at 0 are all equal to that of $g$. In other words, the $C^{\infty}$ topology does not see the germ at 0 while this germ precisely determines the smoothness of the Szekeres vector field and hence the nature of the centralizers in the groups $D^{r}$ for $r \geq 2$. So we do not claim that the phenomenon described in Theorem A is generic in any way, but the following result shows that it is at least not scarce:

Theorem B. Let $f_{0}$ be a smooth contracting diffeomorphism of $\mathbb{R}_{+}$having a smooth and $C^{1}$-bounded Szekeres vector field, and satisfying the following oscillation condition:

$$
\limsup _{x \rightarrow 0}\left(\sup _{0<y \leq x} \frac{\left|\log \left(x-f_{0}(x)\right)\right|}{\left|\log \left(y-f_{0}(y)\right)\right|}\right)=+\infty .
$$

Then, for every $k \geq 0$ and every $\varepsilon>0$, there exists a smooth diffeomorphism $f$ of $\mathbb{R}_{+}$which is close to $f_{0}$ in the sense that

$$
\left|D^{m}\left(f-f_{0}\right)(x)\right| \leq \varepsilon\left|D^{m}\left(f_{0}-\mathrm{id}\right)(x)\right| \text { for all } m \leq k \text { and all } x \in \mathbb{R}_{+},
$$

and whose centralizer $Z_{f}^{\infty}$ is a proper, dense and uncountable subgroup of $Z_{f}^{1}$.

Note that the oscillation condition (19) forces $f_{0}$ to be infinitely tangent to the identity at 0 .

It is interesting to compare this result with Theorem 3.1 in [6]. Indeed, the latter says that, if a smooth contracting diffeomorphism $f$ does not oscillate much in the sense that

$$
\sup _{0<y \leq x}(y-f(y))=\mathrm{O}\left((x-f(x))^{\lambda}\right) \quad \text { for some } \lambda>\frac{r-1}{r},
$$

then the Szekeres vector field of $f$ is $C^{r}$. Theorem B can be thought of as a kind of "partial converse".

Proof. The idea of the proof is the same as for Theorem A: we start with a smooth vector field, here the Szekeres vector field $\xi_{0}$ of the given $f_{0}$ instead of Sergeraert's vector field, and construct deformations $\xi_{k}$ of $\xi_{0}$ which converge to the Szekeres vector field $\xi$ of the wanted $f$. We will just hint at how to adapt the arguments in this more general setting. As before, we denote by $f_{0}^{t}$ the flow of $\xi_{0}$ (so that $f_{0}=f_{0}^{1}$ ) and by $\psi$ the diffeomorphism from $\mathbb{R}$ to $\mathbb{R}_{+}^{*}$ given by $\psi(t)=f_{0}^{t}(1)$ for all $t \in \mathbb{R}$. 
We also fix a forward orbit of $f_{0}$, namely $\left\{a_{l}=f_{0}^{l}(1)=\psi(l), l \geq 0\right\}$, and we set $V_{l}=\left[a_{l+2}, a_{l-2}\right]$ for all $l \geq 0$.

Lemma 5. There exist two alternating sequences of integers $i(n)$ and $j(n), n \geq 0$, with $i(n)<j(n)<i(n+1)<j(n+1)<\cdots$, such that

$$
\frac{\log u_{n}}{\log v_{n}} \underset{n \rightarrow \infty}{\longrightarrow}+\infty
$$

where $u_{n}=\sup _{V_{i(n)}}\left|\xi_{0}\right|$ and $v_{n}=\inf _{V_{j(n)}}\left|\xi_{0}\right|$. In particular, $V_{i(n)}$ and $V_{j(n)}$ are disjoint when $n$ is large enough.

Proof of lemma 5. This proof is rather elementary. Still we give it for the reader's convenience. The oscillation property (19) means that there exist decreasing sequences $\left(x_{n}\right)_{n}$ and $\left(y_{n}\right)_{n}$ converging to 0 , with $y_{n}<x_{n}$, satisfying

$$
\lim _{n \rightarrow \infty} \frac{\log \left(x_{n}-f_{0}\left(x_{n}\right)\right)}{\log \left(y_{n}-f_{0}\left(y_{n}\right)\right)}=+\infty
$$

(the numerator and denominator are negative when $n$ is large enough). We can assume in addition that $x_{n+1} \leq f_{0}^{2}\left(y_{n}\right)$ for all $n$. Let

$$
\begin{aligned}
& i(n)=\max \left\{k \in \mathbb{N}, a_{k} \geq x_{n}\right\}, \\
& j(n)=\min \left\{k \in \mathbb{N}, a_{k} \leq y_{n}\right\} .
\end{aligned}
$$

Any fundamental interval $\left(f_{0}(x), x\right] \subset[0,1]$ of $f_{0}$ contains exactly one element of the forward orbit $\left\{a_{i}, i \in \mathbb{N}\right\}$ of $a_{0}=1$, so the definitions of $i(n)$ and $j(n)$ imply

$$
x_{n+1} \leq a_{i(n+1)}<f_{0}^{-1}\left(x_{n+1}\right) \leq f_{0}\left(y_{n}\right)<a_{j(n)} \leq y_{n}<x_{n} \leq a_{i(n)},
$$

and a fortiori $i(n)<j(n)<i(n+1)$ for all $n$. Let us now prove that for this choice of alternating sequences $i(n)$ and $j(n)$,

$$
\frac{\log u_{n}}{\log v_{n}} \underset{n \rightarrow \infty}{\longrightarrow}+\infty
$$

where $u_{n}=\sup _{V_{i(n)}}\left|\xi_{0}\right|$ and $v_{n}=\inf _{V_{j(n)}}\left|\xi_{0}\right|$. By definition of $i(n), j(n), V_{i(n)}$ and $V_{j(n)}$, there exist $t_{n}$ and $s_{n}$ in $[-3,3]$ such that

$$
u_{n}=\left|\xi_{0}\left(f_{0}^{t_{n}}\left(x_{n}\right)\right)\right| \quad \text { and } \quad v_{n}=\left|\xi_{0}\left(f_{0}^{s_{n}}\left(y_{n}\right)\right)\right|
$$

Now

$$
\frac{d}{d t} f_{0}^{t}(x)=\xi_{0}\left(f_{0}^{t}(x)\right) \quad \text { for all }(t, x) \in \mathbb{R} \times \mathbb{R}_{+},
$$


So

$$
\begin{aligned}
x_{n}-f_{0}\left(x_{n}\right) & =f_{0}^{0}\left(x_{n}\right)-f_{0}^{1}\left(x_{n}\right) \\
& =-\xi_{0}\left(f_{0}^{\tau_{n}}\left(x_{n}\right)\right)=\left|\xi_{0}\left(f_{0}^{\tau_{n}}\left(x_{n}\right)\right)\right| \quad \text { for some } \tau_{n} \in[0,1],
\end{aligned}
$$

and similarly

$$
y_{n}-f_{0}\left(y_{n}\right)=-\xi_{0}\left(f_{0}^{\sigma_{n}}\left(y_{n}\right)\right)=\left|\xi_{0}\left(f_{0}^{\sigma_{n}}\left(y_{n}\right)\right)\right| \quad \text { for some } \sigma_{n} \in[0,1] \text {. }
$$

So

$$
\frac{\log u_{n}}{\log v_{n}}=\frac{\log \left|\xi_{0}\left(f_{0}^{t_{n}}\left(x_{n}\right)\right)\right|}{\log \left|\xi_{0}\left(f_{0}^{s_{n}}\left(y_{n}\right)\right)\right|}=\frac{\log \left(x_{n}-f_{0}\left(x_{n}\right)\right)+\log \left|\frac{\xi_{0}\left(f_{0}^{t_{n}}\left(x_{n}\right)\right)}{\xi_{0}\left(f_{0}^{\tau_{n}}\left(x_{n}\right)\right)}\right|}{\log \left(y_{n}-f_{0}\left(y_{n}\right)\right)+\log \left|\frac{\xi_{0}\left(f_{0}^{s_{n}}\left(y_{n}\right)\right)}{\xi_{0}\left(f_{0}^{\sigma n}\left(y_{n}\right)\right)}\right|} .
$$

The flow $\left(f_{0}^{t}\right)_{t \in \mathbb{R}}$ of $\xi_{0}$ preserves $\xi_{0}$, i.e.

$$
\xi_{0}\left(f_{0}^{t}(x)\right)=D f_{0}^{t}(x) \xi_{0}(x) \quad \text { for all }(t, x) \in \mathbb{R} \times \mathbb{R}_{+} .
$$

As a result,

$$
\frac{\xi_{0}\left(f_{0}^{t_{n}}\left(x_{n}\right)\right)}{\xi_{0}\left(f_{0}^{\tau_{n}}\left(x_{n}\right)\right)}=D\left(f_{0}^{\left(t_{n}-\tau_{n}\right)}\right)\left(f_{0}^{\tau_{n}}\left(x_{n}\right)\right)
$$

and

$$
\frac{\xi_{0}\left(f_{0}^{s_{n}}\left(y_{n}\right)\right)}{\xi_{0}\left(f_{0}^{\sigma_{n}}\left(y_{n}\right)\right)}=D\left(f_{0}^{\left(s_{n}-\sigma_{n}\right)}\right)\left(f_{0}^{\sigma_{n}}\left(y_{n}\right)\right) .
$$

One easily checks that

$$
D f_{0}^{t}(0)=e^{t D \xi_{0}(0)} \quad \text { for all } t \in \mathbb{R},
$$

so since $D f_{0}^{1}(0)=1\left(f_{0}^{1}=f_{0}\right.$ has to be infinitely tangent to the identity at 0 to satisfy the oscillation condition (19)), $D \xi_{0}(0)=0$ and

$$
D f_{0}^{t}(0)=1 \quad \text { for all } t \in \mathbb{R} \text {. }
$$

Since $\xi_{0}$ is $C^{1}$ on $\mathbb{R}_{+}$, both $(t, x) \mapsto f_{0}^{t}(x)$ and $(t, x) \mapsto D f_{0}^{t}(x)$ are uniformly continuous on every compact subset of $\mathbb{R} \times \mathbb{R}_{+}$. Thus, since $\left|\tau_{n}\right| \leq 1,\left|\sigma_{n}\right| \leq 1$, $\left|t_{n}-\tau_{n}\right| \leq 4,\left|s_{n}-\sigma_{n}\right| \leq 4$, and $x_{n}$ and $y_{n}$ converge to 0 ,

$$
D\left(f_{0}^{\left(t_{n}-\tau_{n}\right)}\right)\left(f_{0}^{\tau_{n}}\left(x_{n}\right)\right) \rightarrow 1 \quad \text { and } \quad D\left(f_{0}^{\left(s_{n}-\sigma_{n}\right)}\right)\left(f_{0}^{\sigma_{n}}\left(y_{n}\right)\right) \rightarrow 1 .
$$

This, together with (22), (23), and the fact that

$$
\log \left(x_{n}-f_{0}\left(x_{n}\right)\right) \rightarrow-\infty \quad \text { and } \quad \log \left(y_{n}-f_{0}\left(y_{n}\right)\right) \rightarrow-\infty
$$

implies that

which concludes the proof.

$$
\frac{\log u_{n}}{\log v_{n}} \rightarrow+\infty
$$


We now choose a sequence $w_{n}$ with intermediate decay, i.e. satisfying $w_{n}=$ $\mathrm{o}\left(v_{n}^{m}\right)$ for all $m$ and $u_{n}=\mathrm{o}\left(w_{n}\right)$ (one can take for instance $w_{n}=\sqrt{u_{n}}$ ). Then we define the maps $\gamma_{q, n}$ and $\varphi_{q, n}$ by formulae (11) and (12), using the same function $\gamma$ but the new parameters $w_{n}$ and $j(n)$. Extending thence all other definitions and notation of Subsection 4, our task is to show that Lemmas 3 and 4 still hold.

Proof of Lemma 3 in the general setting. We only insist here on the points that differ from the proof in Subsection 4. Again, we proceed by induction. At step $k$, the choice of $q_{k}$ is just the same, but we need to be more careful about $n_{k}$. The reason is that the map $\psi$ is no longer affine on the regions we consider, and hence the computation of higher derivatives of compositions is trickier.

First, using the fact that $\xi_{0}$ is smooth and infinitely flat at 0 , one can check that, for any fixed $m \geq 1$,

and

$$
\sup \left\{\left|D^{m} \psi(t)\right|, t \in[j(n)-1, \infty)\right\} \underset{n \rightarrow \infty}{\longrightarrow} 0
$$

$$
v_{n}^{m+1} \sup \left\{\left|D^{m} \psi^{-1}(x)\right|, x \in\left[a_{j(n)+1}, a_{j(n)-1}\right]\right\} \underset{n \rightarrow \infty}{\longrightarrow} 0 .
$$

(this is derived from the relations $D \psi=\xi_{0} \circ \psi$ and $D \psi^{-1}=1 / \xi_{0}$ ).

Then we pick an integer $n_{k}>n_{k-1}$ meeting the following three conditions:

$$
\begin{gathered}
\left\|D \psi_{\mid\left[j\left(n_{k}\right)-1, \infty\right)}\right\|_{k-1}<1, \\
\left\|D^{m} \psi_{\mid\left[a_{j\left(n_{k}\right)+1}, a_{j\left(n_{k}\right)-1}\right]}\right\|_{0}<v_{n_{k}}^{-m-1} \text { for } 1 \leq m \leq k,
\end{gathered}
$$

and

$$
\left\|\gamma_{k}\right\|_{k+1} \leq \frac{2^{-k^{2}-4} v_{n_{k}}^{2 k}}{\left|\Pi_{k+1}\right|^{2}\left\|D \Phi_{k-1}\right\|_{k}^{k+1}\left\|\xi_{0}\right\|_{1}}
$$

Inequality (26) is stronger than (15) and thus implies $\left(\mathrm{i}_{k}\right)$ and $\left(\mathrm{ii}_{k}\right)$ of Lemma 3 (the arguments are strictly the same). The proof of ( iii $\left._{k}\right)$ is more complicated but we still have (with our former notation)

$$
f_{k}^{t}=\psi \circ \Phi_{k-1}^{-1} \circ \sigma_{k}^{t} \circ \Phi_{k-1} \circ \psi^{-1} \quad \text { and } \quad f_{k-1}^{t}=\psi \circ \Phi_{k-1}^{-1} \circ \sigma_{0}^{t} \circ \Phi_{k-1} \circ \psi^{-1} \text {. }
$$

For $t=p / q_{k}, 0 \leq p \leq q_{k}$, again $\sigma_{k}^{t}=\sigma_{0}^{t}$ outside

$$
M_{k}=\left[j\left(n_{k}\right)-1+\frac{1}{2 q_{k}}, j\left(n_{k}\right)+\frac{1}{2 q_{k}}\right] \text {, }
$$

so $f_{k}^{t}-f_{k-1}^{t}=0$ outside $\psi\left(M_{k}\right)$. Furthermore, $\Phi_{k-1}=$ id on $M_{k}$. Thus, on $\psi\left(M_{k}\right)$,

$$
f_{k}^{t}=\psi \circ \sigma_{k}^{t} \circ \psi^{-1} \quad \text { and } \quad f_{k-1}^{t}=\psi \circ \sigma_{0}^{t} \circ \psi^{-1}
$$


or, equivalently,

$$
f_{k}^{t}-f_{k-1}^{t}=\left(\psi \circ \sigma_{k}^{t}\right) \circ \psi^{-1}-\left(\psi \circ \sigma_{0}^{t}\right) \circ \psi^{-1} .
$$

For $m \leq k$, Faà di Bruno's formula gives

$$
D^{m}\left(f_{k}^{t}-f_{k-1}^{t}\right)=\sum_{\pi \in \Pi_{m}} D^{|\pi|}\left(\psi \circ \sigma_{k}^{t}-\psi \circ \sigma_{0}^{t}\right) \circ \psi^{-1} \cdot \prod_{B \in \pi} D^{|B|} \psi^{-1} .
$$

According to inequality (25),

$$
\left|\prod_{B \in \pi} D^{|B|} \psi^{-1}\right|<v_{n_{k}}^{-2 k} \quad \text { on } \quad \psi\left(M_{k}\right) \subset\left[a_{j\left(n_{k}\right)+1}, a_{j\left(n_{k}\right)-1}\right] .
$$

Now write

$$
\psi \circ \sigma_{k}^{t}-\psi \circ \sigma_{0}^{t}=\left(\psi \circ \sigma_{0}^{t}\right) \circ\left(\sigma_{0}^{-t} \circ \sigma_{k}^{t}\right)-\left(\psi \circ \sigma_{0}^{t}\right)
$$

and observe, using (17), that

$$
\sigma_{0}^{-t} \circ \sigma_{k}^{t}=\mathrm{id}+\sum_{q=0}^{p-1} \gamma_{k}\left(\mathrm{id}+\frac{q}{q_{k}}\right) \text { for } t=\frac{p}{q_{k}}, 0 \leq p \leq q_{k} .
$$

For $l \leq k$, Faà di Bruno's formula gives

$$
\begin{aligned}
D^{l}\left(\psi \circ \sigma_{k}^{t}-\psi \circ \sigma_{0}^{t}\right) & =D^{l}\left(\left(\psi \circ \sigma_{0}^{t}\right) \circ\left(\sigma_{0}^{-t} \circ \sigma_{k}^{t}\right)-\left(\psi \circ \sigma_{0}^{t}\right)\right) \\
& =\sum_{\substack{\pi \in \Pi_{l} \\
|\pi|<l}} D^{|\pi|}\left(\psi \circ \sigma_{0}^{t}\right) \circ\left(\sigma_{0}^{-t} \circ \sigma_{k}^{t}\right) \cdot \prod_{B \in \pi} D^{|B|}\left(\sigma_{0}^{-t} \circ \sigma_{k}^{t}\right) .
\end{aligned}
$$

Since $\sigma_{0}^{t}=\mathrm{id}+t$, it follows from (24) that

$$
\left|D^{|\pi|}\left(\psi \circ \sigma_{0}^{t}\right) \circ\left(\sigma_{0}^{-t} \circ \sigma_{k}^{t}\right)\right|<1 \quad \text { on } M_{k} .
$$

Now for any partition $\pi \in \Pi_{l}$ with less than $l$ blocks, i.e. $|\pi|<l$, one block $B$ of $\pi$ has at least two elements, so at least one factor in the product

$$
\prod_{B \in \pi} D^{|B|}\left(\sigma_{0}^{-t} \circ \sigma_{k}^{t}\right)=\prod_{B \in \pi} D^{|B|}\left(\mathrm{id}+\sum_{q=0}^{p-1} \gamma_{k}\left(\mathrm{id}+\frac{q}{q_{k}}\right)\right)
$$

is a derivative of order at least 2 , and hence is bounded above by $\left\|\gamma_{k}\right\|_{k}$, while the others are all less than 2 . So the product is bounded above by $2^{l-2}\left\|\gamma_{k}\right\|_{k} \leq$ $2^{k-2}\left\|\gamma_{k}\right\|_{k}$. Therefore,

$$
\left|D^{l}\left(\psi \circ \sigma_{k}^{t}-\psi \circ \sigma_{0}^{t}\right)\right| \leq\left|\Pi_{l}\right| 2^{l-2}\left\|\gamma_{k}\right\|_{k} \leq\left|\Pi_{k}\right| 2^{k-2}\left\|\gamma_{k}\right\|_{k} .
$$

In view of (27), (28) and (26) this implies that $\left\|f_{k}^{t}-f_{k-1}^{t}\right\|_{k} \leq 2^{-k-4}$ for all $t=p / q_{k}, 0 \leq p \leq q_{k}$, and one completes the proof of Lemma 3 just as in Subsection 4. 
Proof of Lemma 4 in the general setting. The proof that the vector fields $\xi_{k}$ converge and that the limit flow $f^{t}$ is smooth for $t \in \mathbb{Z} \oplus \sum_{\tau \in K} \tau \mathbb{Z}$ is strictly the same as in Subsection 5. Note that if we start our construction at step $k_{0}$ instead of step 1, the limit diffeomorphism $f$ satisfies the condition (20) for $l \leq k_{0}$ and $\varepsilon=2^{-k_{0}-1}$, so one can construct $f$ arbitrarily close to $f_{0}$ in the sense of Theorem B.

The part of Lemma 4 that needs a little extra effort is the irregularity of $f^{1 / 2}$. Again,

$$
f^{1 / 2}=\psi \circ \sigma \circ \psi^{-1},
$$

with $\sigma=\Phi^{-1} \circ(\mathrm{id}+1 / 2) \circ \Phi$. The computation of $L \sigma\left(i\left(n_{l}\right)\right)$ leading to (18) can be integrally transposed here, and yields $L \sigma\left(i\left(n_{l}\right)\right)=\sum_{k \geq l} w_{n_{k}} q_{k}^{2}$ (with the new $w_{n}$ ). However, this time $\psi$ is not affine on the involved region, so the computation of $L f^{1 / 2}\left(i\left(n_{l}\right)\right)$ is a bit longer. Formula (2) applied twice gives

$$
L f^{1 / 2}=\left(L \psi \circ\left(\sigma \circ \psi^{-1}\right) \cdot D\left(\sigma \circ \psi^{-1}\right)\right)+\left(L \sigma \circ \psi^{-1} \cdot D \psi^{-1}\right)+L \psi^{-1},
$$

and hence, since $D \psi^{-1}=1 / \xi_{0}$,

$$
L f^{1 / 2}\left(a_{i\left(n_{l}\right)}\right)=\left[L \psi \circ\left(\sigma \circ \psi^{-1}\right) \cdot D\left(\sigma \circ \psi^{-1}\right)+L \psi^{-1}\right]\left(a_{i\left(n_{l}\right)}\right)+\frac{L \sigma\left(i\left(n_{l}\right)\right)}{\xi_{0}\left(a_{i\left(n_{l}\right)}\right)} .
$$

Now, according to Lemma 2 (still valid in our new setting), the limit $\Phi$ of the diffeomorphisms $\Phi_{k}$ coincides with the translation by $1 / 2$ at order one on $\mathbb{Z}$, so the first term of the above sum is equal to

$$
\left[L \psi \circ\left(\mathrm{id}+\frac{1}{2}\right) \circ \psi^{-1} \cdot D\left(\left(\mathrm{id}+\frac{1}{2}\right) \circ \psi^{-1}\right)+L \psi^{-1}\right]\left(a_{i\left(n_{l}\right)}\right)=L f_{0}^{1 / 2}\left(a_{i\left(n_{l}\right)}\right) .
$$

But when $l$ grows, $L f_{0}^{1 / 2}\left(a_{i\left(n_{l}\right)}\right)$ tends to $L f_{0}^{1 / 2}(0)=0$. Therefore

$$
L f^{1 / 2}\left(a_{i\left(n_{l}\right)}\right) \sim \frac{\sum_{k \geq l} w_{n_{k}} q_{k}^{2}}{\xi_{0}\left(a_{i\left(n_{l}\right)}\right)}<-\frac{w_{n_{l}}}{u_{n_{l}}} \underset{l \rightarrow \infty}{\longrightarrow}-\infty,
$$

so $f^{1 / 2}$ is not $C^{2}$ at 0 . This concludes the proof of Lemma 4 and of Theorem B.

\section{References}

[1] D. V. Anosov and A. B. Katok, New examples in smooth ergodic theory. Ergodic diffeomorphisms. Trans. Moscow Math. Soc. 23 (1970), 1-35. Zbl 0255.58007 MR 0370662 416 
[2] H. Eynard, Sur deux questions connexes de connexité concernant les feuilletages et leurs holonomies. PhD Thesis, École Normale Supérieure de Lyon 2009. 415, 416

[3] B. Fayad and A. B. Katok, Constructions in elliptic dynamics. Ergodic Theory Dynam. Systems 24 (2005), no. 5, 1477-1520. Zbl 1089.37012 MR 2104594 416, 421

[4] N. Kopell, Commuting diffeomorphisms. In Global Analysis, Proc. Sympos. Pure Math. XIV, Amer. Math. Soc., Providence, R.I., 1968, 165-184. Zbl 0225.57020 MR 0270396 415,428

[5] A. Navas, Groups of circle diffeomorphisms. arXiv:math/0607481v3 [math.DS] 415

[6] F. Sergeraert, Feuilletages et difféomorphismes infiniment tangents à l'identité. Invent. Math. 39 (1977), 253-275. Zbl 0327.58004 MR 0474327 415, 416, 417, 420, 429

[7] G. Szekeres, Regular iteration of real and complex functions. Acta Math. 100 (1958), 203-258. Zbl 0145.07903 MR 0107016 415, 428

[8] F. Takens, Normal forms for certain singularities of vector fields. Ann. Inst. Fourier 23 (1973), 163-195. Zbl 0266.34046 MR 0365620 415, 429

[9] J.-C. Yoccoz, Centralisateurs et conjugaison différentiable des difféomorphismes du cercle. Astérisque 231 (1995), 89-242. Zbl 0836.30001 MR 1367354415

Received November 10, 2008

Hélène Eynard, UMPA (ENS Lyon), 46, allée d'Italie, 69364 Lyon cedex 7, France

E-mail: heynardb@umpa.ens-lyon.fr 